\title{
SINGULARITY-FREE REGIONS FOR SOLUTIONS OF SYSTEMS OF NONLINEAR COMPLEX DIFFERENTIAL EQUATIONS
}

DAVID V. V. WEND

1. Introduction. This paper is concerned with solutions in the complex plane of the system

$$
y_{k}^{\prime}=f_{k}\left(z, y_{1}, \cdots, y_{n}\right), \quad k=1, \cdots, n,
$$

where for each $k, k=1, \cdots, n, f_{k}$ is regular in $z$ for $|z|<R$ and entire in $y_{1}, \cdots, y_{n}$.

A region $S$ is said to be singularity-free for a solution $\left(y_{1}, \cdots, y_{n}\right)$ if each of $y_{1}, \cdots, y_{n}$ is regular-analytic in $S$. In this paper conditions are obtained under which the system (1.1) has solutions satisfying given initial conditions which are regular in a region which contains a set which is star-like with respect to the origin.

The principal result, Theorem 2.1, compares the growth of the norms of such solutions along each ray emanating from the origin with the growth of solutions of associated real differential equations. Analogous results are obtained for the scalar equation

$$
y^{(n)}+F\left(z, y, y^{\prime}, \cdots, y^{(n-1)}\right)=0 .
$$

These results generalize a theorem obtained by K. M. Das [1] for the equation $y^{\prime \prime}+F(y, z)=0$ and extend results of V. Lakshmikan tham [4] to the complex plane. Essentially the same bounds as those found in $\S \S 2$ and 5 have been obtained independently by K. M. Das for the case $n=2$ [Proc. Amer. Math. Soc. 18 (1967), 220-225].

2. Singularity-free regions for solutions of (1.1). Let $y=\left(y_{1}, y_{2}, \cdots, y_{n}\right), f=\left(f_{1}, f_{2}, \cdots, f_{n}\right)$ and $\alpha=\left(\alpha_{1}, \alpha_{2}, \cdots, \alpha_{n}\right)$. Then (1.1), with given initial values $y_{k}(0)=\alpha_{k}, k=1, \cdots, n$, may be written as

$$
y^{\prime}=f(z, y), \quad y(0)=\alpha .
$$

Let $\|y\|$ be any norm of $y$, for example $\|y\|=\sum_{k=1}^{n}\left|y_{k}\right|,\|y\|$ $=\max \left|y_{k}\right|, k=1, \cdots, n$, or $\|y\|=\left(\sum_{k=1}^{n}\left|y_{k}\right|^{2}\right)^{1 / 2}$.

Let $z=r e^{i \theta}, \theta$ fixed, $0 \leqq \theta<2 \pi$, and assume

$$
\|f(z, y)\| \leqq g_{\theta}(|z|,\|y\|)
$$

Presented to the Society, September 1, 1966 under the title The growth of solutions of systems of nonlinear complex differential equations; received by the editors July 28 , 1966. 
for $|z|<R$ and all $y$, where $g_{\theta}(t, s)$ is continuous for $0 \leqq t<R$ and $0 \leqq s<\infty$. There exists $[2$, p. 25 , Theorem 2.1] a maximal solution $v_{\theta}(x)$ of

$$
u^{\prime}=g_{\theta}(x, u), \quad u(0)=a>0,
$$

defined on an interval $\left[0, r(\theta)\right.$ ), where $r(\theta) \leqq R$ and $v_{\theta}(x) \rightarrow \infty$ as $x \rightarrow r(\theta)$ if $r(\theta)<R$, i.e., $r(\theta)$ is maximal in the interval $[0, R)$.

THEOREM 2.1. Suppose the function $f$ in (2.1) satisfies the inequality (2.2) for each $\theta, 0 \leqq \theta<2 \pi$. Then any solution of $y^{\prime}=f(z, y)$ such that $0<\|y(0)\| \leqq a$ is regular in a region which contains the set $U E_{\theta}$, where $\theta \in[0,2 \pi)$ and $E_{\theta}=\left\{z \mid z=r e^{i \theta}\right.$ and $\left.0 \leqq r<r(\theta)\right\}$. Furthermore $\left\|y\left(r e^{i \theta}\right)\right\|$ $\leqq v_{\theta}(r)$ on each ray $z=r e^{i \theta}, 0 \leqq r<r(\theta), v_{\theta}$ and $r(\theta)$ defined above.

Proof. Fix $\theta, 0 \leqq \theta<2 \pi$, and let $z=r e^{i \theta}$. Let $w(r)=\left\|y\left(r e^{i \theta}\right)\right\|$, where $y(z)$ is a solution of $(2.1)$ such that $0<\|y(0)\| \leqq a$. Then for $h>0$

$$
\frac{1}{h}[w(r+h)-w(r)] \leqq\left\|\frac{y\left((r+h) e^{i \theta}\right)-y\left(r e^{i \theta}\right)}{h}\right\|,
$$

so

$$
D^{+} w(r) \leqq\left\|\frac{\partial y}{\partial r}\left(r e^{i \theta}\right)\right\|=\left\|\frac{d y}{d z} e^{i \theta}\right\|=\left\|\frac{d y}{d z}\right\|=\|f(z, y)\| \leqq g_{\theta}(r, w(r)),
$$

where $D^{+} w$ is the upper right-hand derivative of $w$. Thus $D^{+} w(r)$ $\leqq g_{\theta}(r, w(r)), w(0) \leqq v_{\theta}(0)$, so $[2$, p. 26 , Theorem 4.1 and p. 27 , Remark 2] $w(r)=\left\|y\left(r e^{i \theta}\right)\right\| \leqq v_{\theta}(r)$ on $[0, r(\theta))$. Therefore $y$ is regular for $z=r e^{i \theta}, 0 \leqq r<r(\theta), 0 \leqq \theta<2 \pi$.

Corollary 2.2. Suppose $f$ satisfies (2.2). If solutions of (2.3) are unique and there exist continuously differentiable functions $u_{\theta}(x)$ on $[0, s(\theta)), 0<s(\theta) \leqq R, 0 \leqq \theta<2 \pi$, such that for each $\theta$

$$
u_{\theta}^{\prime}(x) \geqq g_{\theta}\left(x, u_{\theta}\right), \quad u_{\theta}(0)=a,
$$

on $[0, s(\theta))$, then any solution of (2.1) for which $\|y(0)\| \leqq a$ is regular in a region containing the set $\cup E_{\theta}$, where $\theta \in[0,2 \pi)$ and $E_{\theta}=\left\{z \mid z=r e^{i \theta}\right.$ and $0 \leqq r<s(\theta)\}$.

Note. This corollary is our generalization of [1, Theorem 2.1].

Proof. From [2, p. 26, Remark 1] it follows that $s(\theta) \leqq r(\theta)$ for each $\theta$.

REMARKs. 1. If (2.3) does not have a unique solution, then it is not certain that the conclusion of Corollary 2.2 is valid. For example, with $g_{\theta}(x, u)=(u-1)^{2 / 3} \exp (u-1)$ for each $\theta$ and $u(0)=1$, equation (2.3) becomes 


$$
u^{\prime}=(u-1)^{2 / 3} \exp (u-1), \quad u(0)=1,
$$

which has one solution $u(x)=1$ and a maximal solution $v$ defined by

$$
x=\int_{0}^{v-1} t^{-2 / 3} e^{-t} d t .
$$

As $v \rightarrow \infty, x \rightarrow r_{0}=\int_{0}^{\infty} t^{-2 / 3} e^{-t} d t<\infty$. With equality in (2.4) and $u_{\theta}(0)=1$, $s(\theta)$ may be chosen for each $\theta$ to be greater than $r_{0}=r(\theta)$.

2. A function $g_{\theta}$ such as the one in (2.2) always exists: for example, $\|f(z, y)\| \leqq g_{\theta}(|z|,\|y\|)=\max \|f(t, s)\|$ on the domain $I \times I^{\prime}$, where $I=I(|z|)=\{t|0 \leqq| t|\leqq| z \mid\}$ and $I^{\prime}=I^{\prime}(\|y\|)=\{s \mid 0 \leqq\|s\| \leqq\|y\|\}$. Here the function $g_{\theta}(t, s)=g(t, s)$ is nondecreasing in both $t$ and $s$. In this case it can be shown that the system (2.3) has unique solutions if $g(x, u(0))>0$ for $x>0$. (The example in the previous remark shows (2.3) may not have unique solutions if $g(x, u(0))=0$.)

3 . Finally, we note that the choice of norm in (2.2) may influence the choice of the function $g$ and thus the regions in which solutions of (1.1) are guaranteed regular.

3. Nonlinear equations of the $n$th order. In this section we will consider the equation

$$
y^{(n)}+F\left(z, y, y^{\prime}, \cdots, y^{(n-1)}\right)=0 .
$$

Although (3.1) can be transformed to the form (2.1), we will see later by an example in $\$ 4$ that some sharpness may be lost by doing so.

Suppose the function $F$ is regular in $z$ for $|z|<R$ and entire in the remaining $n$ variables. Let

$$
F\left(z, y, \cdots, y^{(n-1)}\right) \leqq G\left(|z|,|y|, \cdots,\left|y^{(n-1)}\right|\right)
$$

for $|z|<R$ and all values of $y, y^{\prime}, \cdots, y^{(n-1)}$, where $G\left(t, s_{1}, \cdots, s_{n}\right)$ is continuous in $t, 0 \leqq t<R$, and continuous and nondecreasing for all nonnegative values of $s_{1}, \cdots, s_{n}$. There exists $[2$, p. 14, Corollary 3.1 and p. 28, Exercise 4.3] a maximal solution $v$ of

$$
u^{(n)}=G\left(x, u, \cdots, u^{(n-1)}\right), \quad u^{(k)}(0)=a_{k} \geqq 0,
$$

$k=0, \cdots, n-1, a_{0}+\cdots+a_{n-1}>0$. defined on a maximal subinterval $\left[0, r_{0}\right)$ of $[0, R)$.

THEOREM 3.1. If the function $F$ in (3.1) satisfies (3.2), then any solution of (3.1) for which $\left|y^{(k)}(0)\right|=a_{k}, k=0, \cdots, n-1$, is regular for $|z|<r_{0}$.

ProOF. If $y(z)$ is a solution of (3.1) such that $\left|y^{(k)}(0)\right|=a_{k}$, $k=0, \cdots, n-1$, then 


$$
\begin{aligned}
y^{(k)}(z)= & \sum_{j=k}^{n-1} \frac{z^{j-k}}{(j-k) !} y^{(j)}(0) \\
& -\int_{0}^{z} \frac{(z-\zeta)^{n-k-1}}{(n-k-1) !} F\left(\zeta, y(\zeta), \cdots, y^{(n-1)}(\zeta)\right) d \zeta,
\end{aligned}
$$

$0 \leqq k \leqq n-1$, where the integral is taken along the segment $\zeta=t e^{i \theta}$, $0 \leqq t \leqq x\left(z=x e^{i \theta}\right)$. Therefore

$$
\begin{aligned}
\left|y^{(k)}(z)\right| \leqq & \sum_{j=k}^{n-1} a_{j} \frac{x^{j-k}}{(j-k) !} \\
& +\int_{0}^{x} \frac{(x-t)^{n-k-1}}{(n-k-1) !} G\left(t,|y(\zeta)|, \cdots,\left|y^{(n-1)}(\zeta)\right|\right) d t .
\end{aligned}
$$

Let $u_{\delta}$ be a solution of (3.3) with $a_{k}$ replaced by $a_{k}+\delta, k=0, \cdots$, $n-1, \delta>0$. Then

$$
\begin{aligned}
u_{\delta}^{(k)}(x)= & \sum_{j=k}^{n-1} \frac{x^{j-k}}{(j-k) !}\left(a_{j}+\delta\right) \\
& +\int_{0}^{x} \frac{(x-t)^{n-k-1}}{(n-k-1) !} G\left(t, u_{\delta}, \cdots, u_{\delta}^{(n-1)}\right) d t,
\end{aligned}
$$

and

$$
\begin{aligned}
u_{\delta}^{(k)}(x)-\left|y^{(k)}(z)\right| \geqq \delta \sum_{j=k}^{n-1} \frac{x^{j-k}}{(j-k) !}+\int_{0}^{x} \frac{(x-t)^{n-k-1}}{(n-k-1) !} \\
\cdot\left\{G\left(t, u_{\delta}, \cdots, u_{\delta}^{(n-1)}\right)-G\left(t,|y|, \cdots,\left|y^{(n-1)}\right|\right)\right\} d t .
\end{aligned}
$$

Since $u_{\delta}^{(k)}(0)>\left|y^{(k)}(0)\right|$ for each $k,\left|y^{(k)}(z)\right|<u_{\delta}^{(k)}(x)$ for all $x \geqq 0$ sufficiently small. Suppose for some $\theta,\left|y^{(k)}(\zeta)\right|<u_{\delta}^{(k)}(t), 0 \leqq t<x$, for each $k$, but for some $k,\left|y^{(k)}(z)\right|=u_{\delta}^{(k)}(x)$. Then it follows from (3.4) that $G\left(t, u_{\delta}, \cdots, u^{(n-1)}\right)-G\left(t,|y|, \cdots,\left|y^{(n-1)}\right|\right)<0$ for some $t$, $0<t<x$, a contradiction, since $G$ is nondecreasing in the last $n$ variables. Therefore $|y(z)|<u_{\delta}(x)$ on $\left[0, r_{\delta}\right)$, where $r_{\delta}$ is the right-hand endpoint of the maximal subinterval of $[0, R)$ on which $u_{\delta}$ is defined. But $\left[2\right.$, p. 14, Theorem 3.2] $u_{\delta} \rightarrow v$ and $r_{\delta} \rightarrow r_{0}$ as $\delta \rightarrow 0$. Therefore $|y(z)|$ $\leqq v(x)$ on $\left[0, r_{0}\right)$, so $y(z)$ is regular in $|z|<r_{0}$.

Analogous to Corollary 2.2 we have

Corollary 3.2. Suppose F satisfies (3.2). If solutions of (3.3) are unique and there exists a continuous function $u(x)$ on $[0, r), r \leqq R$, such that $u^{(k)}(0)=a_{k} \geqq 0, k=0,1, \cdots, n-1, a_{0}+\cdots+a_{n-1}>0$, and $u^{(n)}(x) \geqq G\left(x, u(x), \cdots, u^{(n-1)}(x)\right)$ on $[0, r)$, then any solution of (3.1) for which $\left|y^{(k)}(0)\right|=a_{k}, k=0, \cdots, n-1$, is regular in $|z|<r$. 
REMARKs. 1. An example showing the necessity of the uniqueness hypothesis in our proof of Corollary 3.2 is given by

$$
u^{\prime \prime}=(u-x)^{2 / 3} \exp (u-x), \quad u(0)=0, \quad u^{\prime}(0)=1 .
$$

2. The disks in Theorem 3.1 and Corollary 3.2 can be extended to regions containing sets star-like with respect to the origin, and the requirements $\left|y^{(k)}(0)\right|=a_{k}$ may be replaced by $\left|y^{(k)}(0)\right| \leqq a_{k}$ to yield results similar to those in $\S 2$.

3. An equation which can be reduced to (3.1) but which can be handled independently and may lead to a different $G$ function, and therefore possibly sharper results, is

$$
\left(r(z) y^{(m)}\right)^{(n)}+F\left(z, y, y^{\prime}, \cdots, y^{(m)}\right)=0,
$$

where $r(z)$ is regular and nonzero for $|z|<R$ and $F$ is regular for $|z|<R$ and entire in the remaining $m+1$ variables.

4. An example. Assuming the inequality (3.2) may lead to a somewhat sharper result than assuming the inequality (2.2) for the corresponding system. For example, consider $y^{\prime \prime}=e^{y}, y(0)=1, y^{\prime}(0)=0$. Here $\left|e^{y}\right| \leqq \exp |y|=G(|z|,|y|)$, and the related real equation is $u^{\prime \prime}=e^{u}, u(0)=1, u^{\prime}(0)=0(u=|y|)$. Solving this equation,

$$
r_{0}=\int_{1}^{\infty} \frac{d t}{\left(2 e^{t}-2 e\right)^{1 / 2}}=\pi /(2 e)^{1 / 2} \approx 1.347 .
$$

This value for $r_{0}$ is sharp, i.e. the solution $y(z)$ is not regular in any disk with center at the origin and radius larger than $r_{0}$.

The corresponding vector equation may be written $\left(u_{1}, u_{2}\right)^{\prime}$ $=\left(u_{2}, e^{u_{1}}\right),\left(u_{1}, u_{2}\right)(0)=(1,0)$. Using $\left\|\left(u_{1}, u_{2}\right)\right\|=\left|u_{1}\right|+\left|u_{2}\right|,\left\|\left(u_{2}, e^{u_{1}}\right)\right\|$ $\leqq\left\|\left(u_{1}, u_{2}\right)\right\|+\exp \left\|\left(u_{1}, u_{2}\right)\right\|$, so for the corresponding scalar equation may be chosen $v^{\prime}=v+e^{v}, v(0)=1$. In this case, $r_{0}=\int_{1}^{\infty} d t /\left(t+e^{t}\right) \approx 0.315$, less than the value of $r_{0}$ in (4.1).

It is interesting to compare these results with those obtained by the method of successive approximations [3, p. 71] and the method of limits [3, p. 284]. The method of limits gives the maximum value $1 / 3 e^{2}$ for $r_{0}$, less than either of the values obtained above, while the method of successive approximations gives the maximum value 1 for $r_{0}$, between the two values obtained above.

5. Lower bounds for $\|y(z)\|$. Suppose the function $f$ in (2.1) satisfies (2.2). Let $u_{\theta}$ be the minimal solution of $u^{\prime}=-g_{\theta}(x, u), u(0)=a>0$, defined on a maximal subinterval $[0, r(\theta))$ of $[0, R)$. Then as in $\S 2$ it can be shown $[2$ p. 26, Remark 1] that for solutions of $(2.1)$ such that 
$\|y(0)\| \geqq a,\left\|y\left(x e^{i \theta}\right)\right\| \geqq u_{\theta}(x)$ on $[0, r(\theta))$, of significance up to the first positive zero of $u_{\theta}$.

\section{REFERENCES}

1. K. M. Das, Singularity-free regions for solutions of 2 nd order non-linear differential equations, J. Math. Mech. 13 (1964), 73-84.

2. P. Hartman, Ordinary differential equations, Wiley, New York, 1964.

3. E. L. Ince, Ordinary differential equations, Dover, New York, 1926.

4. V. Lakshmikantham, Upper and lower bounds of the norm of solutions of differ ential equations, Proc. Amer. Math. Soc. 13 (1962), 615-616.

UNIVERSITY OF UTAH AND

Montana State University

\section{ASYMPTOTIC ALMOST PERIODICITY OF SOLUTIONS OF A SYSTEM OF DIFFERENTIAL EQUATIONS}

\section{W. R. UTZ AND PAUL WALTMAN ${ }^{1}$}

The theorems of this paper give sufficient conditions for the asymptotic almost periodicity of bounded solutions of a system of differential equations in the plane. Let $x=\left(x_{1}, x_{2}\right)$ and let

$$
\rho(x, y)=\|x(t)-y(t)\|=\left|x_{1}(t)-y_{1}(t)\right|+\left|x_{2}(t)-y_{2}(t)\right| .
$$

The system to be considered, using this vector agreement, is

$$
x^{\prime}=f(t, x) \text {. }
$$

The theorems that we prove were suggested by a paper of J. S. W. Wong and T. A. Burton [4] who consider the system (1) of the special form

$$
x_{1}^{\prime}=x_{2}, \quad x_{2}^{\prime}=-a(t) f\left(x_{1}\right) g\left(x_{2}\right) .
$$

The proofs of our theorems differ considerably from the proofs of Wong and Burton. An important difference is our use of periodic solutions of a limiting system of (1) to avoid the use of Liapunov functions.

A solution, $x=x(t)$, of (1) valid for all large $t$ is said to be asymptotically almost periodic if there exists a positive real number $T$ such that for every $\epsilon>0$ there corresponds a real number $t(\epsilon)$ for which

Presented to the Society, April 14, 1967; received by the editors August 1, 1966.

1 Research of this author supported by NSF Grant GP-5599. 